\title{
Diseño organizacional mediante el enfoque sistémico y cibernético: El caso de una empresa prestadora de saneamiento
}

\author{
Organizational design through a systemic and cybernetic approach: \\ The case of an enterprise of sanitation \\ Robensoy Marco Taipe Castro ${ }^{1 *}$ \\ Recibido 24 de octubre de 2017, aceptado 08 de octubre de 2018 \\ Received: October 24, 2017 Accepted: October 08, 2018
}

\begin{abstract}
RESUMEN
El caso de estudio es la aplicación de un marco de trabajo basado en una intervención sistémica y la cibernética organizacional, que permita un diseño organizacional de una empresa de saneamiento para su restructuración y reorganización. Es decir, realizar de forma inmediata innovaciones efectivas para el análisis, diagnóstico y diseño organizacional de la empresa de saneamiento. El diseño organizacional evidencia un marco de trabajo de cibernética organizacional y procesos, un diseño de la estructura organizacional (autonomía, balance interno, balance con el entorno, mecanismo de identidad y política), el modelo de sistema viable modo idealizado, funciones sistémicas que contienen macro procesos, procesos y sub procesos, y complementario a ello, el mapa de procesos de la empresa. El caso de estudio contribuye a un proceso particular de aplicación sistémica y cibernética organizacional en el contexto del servicio público de saneamiento local en la mejora de su sistema de gestión.
\end{abstract}

Palabras clave: Cibernética organizacional, Intervención sistémica, Servicio público de saneamiento, diseño organizacional.

\begin{abstract}
The case study is the application of a framework based on a systemic intervention and organizational cybernetics, which allows an organizational design of an enterprise of sanitation for its restructuring and reorganization. That is, to immediately make effective innovations for the analysis, diagnosis and organizational design of the enterprise of sanitation. The organizational design evidences a framework of organizational cybernetics and processes, a design of the organizational structure (autonomy, internal balance, balance with the environment, identity and policy mechanism), the viable system model idealized mode, macro processes, processes and sub processes, and complementary to it, the process map of the enterprise. The case study contributes to a particular process of systemic and organizational cybernetics application in the context of the local public sanitation service in the improvement of its management system.
\end{abstract}

Keywords: Organizational cybernetics, systemic intervention, public sanitation service, organizational design.

\footnotetext{
1 Facultad de Ingeniería de Sistemas, Universidad Nacional del Centro del Perú, Av. Mariscal Castilla No 3909 - El Tambo Huancayo, Perú.

* Autor de correspondencia: rtaipec@ gmail.com
} 


\section{INTRODUCCIÓN}

En el informe de la Comisión Económica para América Latina y el Caribe (CEPAL) sobre los servicios de saneamiento en el Perú, existe una relación directa entre la prevalencia de enfermedades diarreicas y la carencia de servicios de saneamiento en especial en menores de 5 años de edad [1].

En el Perú existen cincuenta empresas prestadoras de servicios de saneamiento (EPS) según la Superintendencia Nacional de Servicios de Saneamiento (SUNASS). Las EPS operan en su mayoría en áreas urbanas, SEDAPAL en Lima, 48 EPS municipales en el resto del país; ellas prestan el servicio a cerca de 19 millones de habitantes. No obstante, su modelo de gestión data de la década de 1960. Las EPS pierden 64\% del agua que producen, debido a su modelo administrativo colapsado por injerencias políticas, la deficiente gestión del recurso humano y la débil supervisión [2].

Por otro lado, el diseño organizacional mediante la sistémica y cibernética ha sido estudiado y tratado por varios autores que han complementado los estudios de cibernética organizacional de Stafford Beer [3, 4]. Algunos de esos trabajos son extensos y nutren la manera de intervenir en las problemáticas complejas-sistémicas de las organizaciones. Intervenir la complejidad a partir de los instrumentos que proporciona la ciencia de la "Kybernetes", que se viene aplicando en los últimos tiempos en las organizaciones [5]. Establecer las implicaciones prácticas de un enfoque sistémico apropiado para la construcción y diseño de organizaciones capaces de sobrevivir y prosperar en estos tiempos turbulentos [6]. Sistemas organizacionales mediante el manejo de la complejidad con el modelo del sistema viable, clarifica la aplicación de ideas cibernéticas -particularmente aquellas del modelo del sistema viable de Stafford Beer- al diagnóstico y diseño de organizaciones de todo tipo [7]. Los aportes han sido muy significativos y de relevancia en el ámbito organizacional [8], presenta cuestiones adicionales y estructuras viables relacionadas de las organizaciones, que se desvían del modelo de Beer. Estas cuestiones son: el establecimiento y la evolución de una organización; sistemas para el control de independiente; sistemas para la corrección independiente de problemas de rendimiento, ambos trabajando fuera de una estructura jerárquica; sistemas de producción de tracción y sistemas de chequeo y equilibrio de poder de nivel superior (como juntas y reuniones de accionistas). Puche, [9], ha demostrado que el enfoque sistémico (holístico), basado en la optimización global, tiene un rendimiento significativamente mejor. Ello se basa en la aplicación combinada del Modelo del Sistema Viable de Beer y la Teoría de las restricciones de Goldratt, también la aplicación del modelo de sistema viable en la organización de proyectos de software [10]. Perez, [11], expone cómo los conceptos relacionados con la cibernética organizacional (OC) podrían ser utilizados en combinación con las tecnologías de la información y la comunicación (TIC) para facilitar las discusiones de grupo sobre temas complejos y para mostrar su impacto en un caso real. El estudio de un marco general de sistemas para una universidad de investigación viable, utiliza el modelo de sistema viable como una herramienta de modelado para desarrollar un marco sistémico para las universidades de investigación viables. Hildbrand, [13], proporciona directrices detalladas sobre cómo llevar a cabo un diagnóstico mediante el modelo de sistema viable de la Cibernética Organizacional junto con métodos de investigación cualitativa, describe la recopilación de datos, el análisis y la presentación de los resultados. Puche, [14], detalla la importancia particular de los Sistemas Uno y Cuatro en un proyecto de software; en otras palabras, los proyectos de software necesitan definir claramente sus elementos operacionales (por ejemplo, unidades organizativas, unidades de negocio, entornos de trabajo y equipos de trabajo) y las relaciones que aparecen entre ellos; además, en los proyectos de software es necesario determinar las acciones de prevención adecuadas para poder observar los cambios que se producen en su entorno y así tomar decisiones que permitan al proyecto adaptarse a estos cambios. Gershenson, [15], al relacionar la autopoiesis con la ley de Ashby de la variedad requerida, la auto-organización se presenta como una forma en la que los sistemas pueden ser diseñados para adaptarse a la variedad de su entorno. Schwaninger, [16], desarrolla una estructura que permite a los agentes de todos los estratos recursivos generar variedad en equilibrio con las complejidades que enfrentan; la arquitectura organizativa basada en el modelo de sistema viable, es aplicada a cada uno de esos niveles, asegura las necesarias y suficientes condiciones estructurales para la sostenibilidad del sistema bajo estudio. 
Espinoza, [17], recurre al modelo de sistema viable como meta-lenguaje para facilitar la sostenibilidad a largo plazo en los negocios, las comunidades y las sociedades, utilizando la "Metodología para apoyar la auto-transformación", centrándose en las formas de aprender sobre la gobernanza para la sostenibilidad. Sagalovsky, [18], aborda las implicaciones organizacionales de las iniciativas de implementación Lean de transformación, aprovechando el modelo de sistema viable para entender lo que se necesita en la organización para que estas iniciativas puedan tener éxito y tomar raíz. Pérez, [19], expone cómo los conceptos relacionados con la cibernética organizacional podrían ser utilizados en combinación con las tecnologías de la información y la comunicación (TIC) para facilitar las discusiones de grupo sobre temas complejos y mostrar su impacto en un caso real. Terra, [20], propone una mejor distribución del poder que podría asegurar una mayor autonomía y estabilidad del gobierno en la república. Esto se logra aplicando los principios del modelo de sistema viable de Stafford Beer a las tres potencias estatales de Brasil: el ejecutivo, el legislativo y el judicial. Parraguez, [21], a la falta de una caracterización sistemática de las interfaces de proceso entre las actividades de diseño de ingeniería, propone un enfoque que caracteriza las interfaces de proceso como redes de organización que consisten en personas y sus interacciones al realizar actividades de interconexión. Además, ofrece orientación sobre cómo probar e interpretar el efecto de esas características en problemas de interfaz. Lowe, [22], describe el desarrollo y la aplicación de un innovador método de estructuración de problemas para orientar las intervenciones en la forma en que el Ministerio de Defensa del Reino Unido ofrece proyectos de infraestructura y servicios; este método utiliza una adaptación del modelo de sistema viable para estructurar un conjunto de evaluaciones de partes interesadas que conduce a la construcción de un Modelo de Proceso Jerárquico. Schwaninger, [23], pauta una forma de probar la teoría empíricamente del modelo de sistema viable, sobre la base de una encuesta amplia y un análisis cuantitativo pertinente, los datos disponibles apoyan las hipótesis y con ello corroboran la teoría del modelo de sistema viable. Walker, [24], describe una serie de intervenciones de transformación que han utilizado los principios del modelo de sistemas viables para mejorar la gobernanza empresarial y hacer evidente la criticidad del funcionamiento eficaz de los Sistemas 2-5 del modelo de sistemas viables; también considera por qué, cuando tales transformaciones individuales han apoyado es evidente la viabilidad de las autoridades locales, el sector aún no ha adoptado el pensamiento sistémico para mejorar sus sistemas empresariales rectores.

Sin embargo, el diseño organizacional aun demanda la intervención y desarrollo de métodos nuevos enfocados en resultados inmediatos y de alto impacto, además, existe poca evidencia en utilizar el enfoque por procesos aunado a la cibernética organizacional en el diseño organizacional.

En el trabajo, el caso de estudio, contribuye en describir y puntualizar el diseño organizacional mediante la cibernética organizacional y procesos de la EPS SEDAM HUANCAYO S.A. La Cibernética Organizacional hace referencia a uno de los enfoques sistémicos, de la "cibernética" de Wiener (1948), que aplica los principios cibernéticos relacionados con la "comunicación y control" a las organizaciones; describe las condiciones necesarias y suficientes para que una organización sea viable (que puede vivir, desarrollarse con autonomía); una organización es viable si cumple con las seis funciones sistémicas, al igual del cerebro y el sistema nerviosos humano, estas son: Operación (Sistema 1), coordinación (Sistema 2), control (Sistema 3), monitoreo-auditoria (Sistema 3*), inteligencia (Sistema 4) y por último identidad (Sistema 5) [4]. En el Enfoque basado en procesos, principio de gestión, asume como "proceso" al conjunto de actividades secuenciales que realizan una transformación de una serie de entradas (material, mano de obra, capital, energía e información) en las salidas deseadas (bienes o servicios) añadiendo valor. La Cibernética Organizacional encamina el sistema organizativo, su diseño o diagnóstico y define las recursiones necesarias para identificar y diseñar los procesos, ello es complementado con el Enfoque basado en procesos al implementar y operar el sistema organizativo.

\section{MARCO METODOLÓGICO DE DISEÑO ORGANIZACIONAL MEDIANTE UNA INTERVENCIÓN SISTÉMICA Y CIBERNÉTICA}

El diseño organizacional evidencia: un marco de trabajo de cibernética organizacional y procesos 
[5], [25], un diseño de la estructura organizacional (autonomía, balance interno, balance con el entorno, mecanismo de identidad y política), el modelo de sistema viable modo idealizado, funciones sistémicas que contienen macro procesos, procesos y sub procesos, y complementario el mapa de procesos de la empresa. Todo ello mediante las siguientes fases:

\section{Diseñar operación y autonomía}

- Se debe establecer y afinar el propósito de la actividad misional, es decir la definición del sistema. (¿Qué hace?, ¿Cómo?, ¿Para qué? Definidos en una oración).

- Listar los componentes de la unidad operacional acorde al propósito identificado. (Subsistemas).

- Identificar a que suprasistema pertenece.

- Integración con el entorno. ¿Cómo se procede a integrar? Establecer el requerimiento y el usuario, así como el resultado y el beneficiario.

- La actividad misional debe contar con un plan de operación, y debe estar sujeto a responsabilidad, negociación de recursos y a reglas de intervención predefinidas. Además, el plan debe estar alineado al propósito de la actividad misional y este a su vez debe estar alineado al propósito general de la organización.

- Se deben establecer los límites de autonomía, en caso de no cumplir el propósito, también la dependencia a la negociación de recursos (es decir si se les provee los recursos necesarios a tiempo), la dependencia con otras unidades operativas (es decir el intercambio de resultados y productos entre unidades operacionales) provocan límites en la autonomía.

- Debe existir una coordinación a nivel local, así como una coordinación a nivel central que interactúe con los miembros de soporte (sistema 3).

- Se debe listar todas las coordinaciones con los miembros de soporte (sistema 3).

- Establecer mecanismos de seguimiento local y también de seguimiento central. (Que verifique como se utilizan los recursos, el personal, etc.). Estos mecanismos no se implantan con la finalidad de sancionar o castigar más bien con el propósito de encontrar indicios de situaciones problemas.

\section{Diseñar el balance interno}

- Negociar los problemas estructurales en las unidades operacionales tanto como sea posible.
- Incrementar las capacidades de cohesión y coordinación para incrementar sus capacidades de negociación.

Diseñar el mecanismo de balance con el entorno

- Identificar el entorno futuro.

- Identificar la interrelación entre la cohesión y adaptación.

- Identificar el monitoreo de la interrelación de la adaptación y la cohesión.

- Identificar el cómo la adaptación se mantiene en contacto con el exterior.

- Identificar las capacidades de adaptación e inteligencia para formular sus planes de la organización con el propósito de adaptarse a los cambios en el entorno.

- Nombrar el rol del sistema 3 y el rol del sistema 5.

\section{Diseñar el mecanismo de identidad y ethos}

- Identificar la relación del sistema 5 con el sistema 4 a través de planes para la formulación.

- Identificar la relación del sistema 5 con el sistema 3 a través de las capacidades de las unidades operacionales y la sinergia generada.

- Identificar quienes deben ser miembros del mecanismo de política.

- Definir el rol de los integrantes de la política.

- Identificar los mecanismos para acceder a la representatividad en el sistema 5.

\section{Complementario a las etapas mencionadas desarrollar las siguientes actividades:}

- Diseño de descentralización y centralización.

- Desplegar las funciones sistémicas versus actividades de regulación.

- Definir macro procesos y procesos acorde a las funciones sistémicas de viabilidad (Misionales, Cohesión, Estabilización y sincronización, auditoria y monitoreo, adaptación e inteligencia, e identidad y política).

- Desarrollar el mapa de procesos de la organización.

\section{RESULTADOS}

En la ciudad de Huancayo la EPS es SEDAM Huancayo S.A., una empresa de Sociedad Anónima, de propiedad de la Municipalidad de Huancayo y las Municipalidades Distritales del Tambo, Chilca, Huancán, Viques y Huacrapuquio, con personería jurídica de derecho público, patrimonio propio, 
autonomía funcional, económica, técnica, financiera y administrativa.

Según sus propios informes estadísticos de gestión, la situación de SEDAM Huancayo S.A. es:

- Insuficiente cobertura de servicios de agua, saneamiento y tratamiento de aguas residuales.

- Mala calidad de la prestación de servicios pone en riesgo la salud de la población.

- Deficiente sostenibilidad de los sistemas construidos.

- Tarifas que no permiten cubrir los costos de inversión, operación y mantenimiento de los servicios (atraso tarifario).

- El tamaño de los mercados bajo responsabilidad de las EPS no garantiza una buena gestión, no permiten economías de escala ni viabilidad financiera.

- Debilidad institucional y financiera.

- Recursos humanos en exceso, poco calificados y con alta rotación en las EPS.

El Informe Final de Diagnóstico de Procesos y Diseño Organizacional de SEDAM-HUANCAYO S.A. 2012, detalla el diagnóstico de autonomía, de coordinación, de cohesión, de monitoreo-auditoria, de adaptación-inteligencia y de identidad-política.
El diseño organizacional de la empresa es orientado a la implementación del propósito: "Brindar el servicio de agua y saneamiento en su ámbito, en equilibrio con el ecosistema".

El inicio del diseño organizacional es el diseño de operación y autonomía de la empresa considerando los siguientes componentes: Producción de agua potable, Comercialización de servicios de saneamiento y por último, Alcantarillado y tratamiento de aguas residuales. Figura 1, Figura 2 y Figura 3.

En seguida, se realiza el diseño del balance interno de la empresa, Figura 4, con la idea de describir la interacción de las unidades misionales u operacionales, tanto como sea posible e incrementar la capacidad de cohesión y coordinación para fortalecer sus capacidades de negociación. Además, los elementos del balance interno, "El aquí y el ahora" (Implementación, Coordinación, Cohesión y Monitoreo) de SEDAM HUANCAYO S.A. son:

- Implementación: Producción de Agua Potable, Comercialización de Servicios de Saneamiento, Tratamiento y Disposición de Aguas Residuales.

- Coordinación: Los Comités de Gestión.

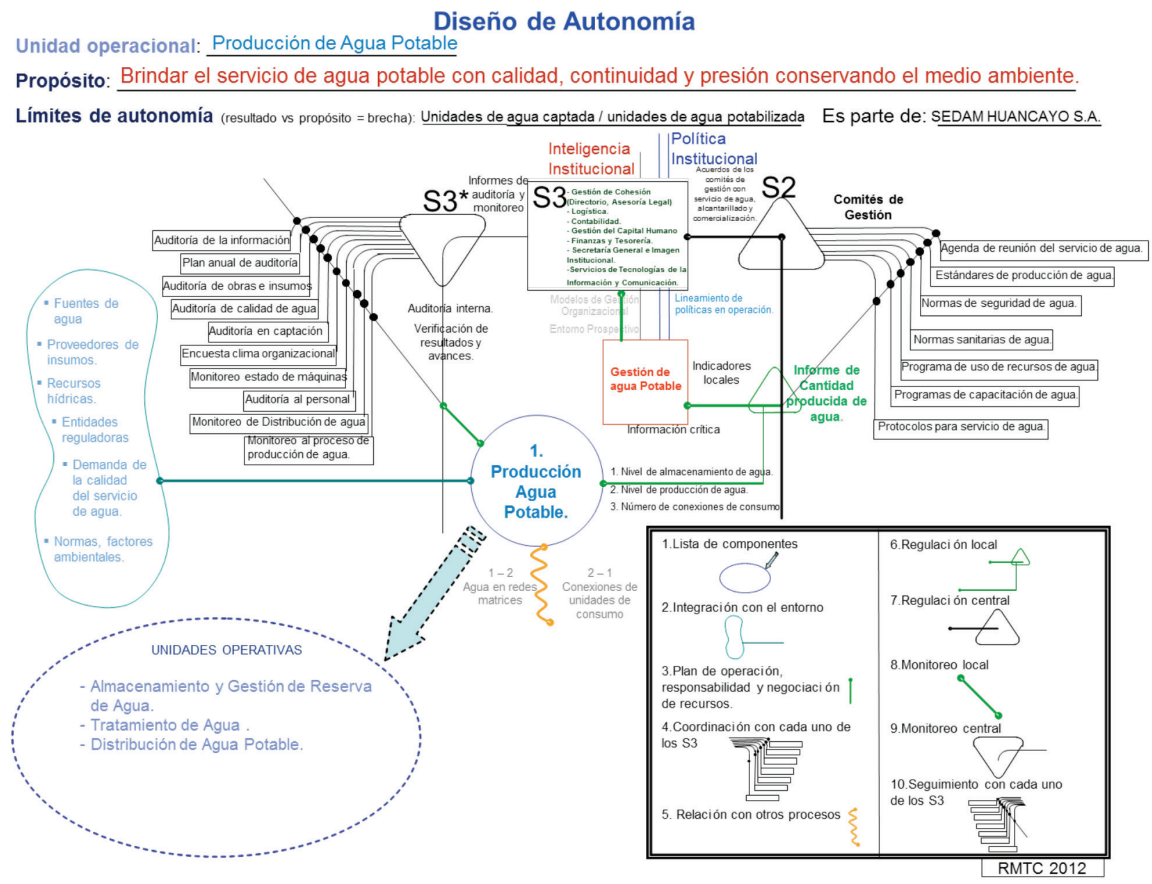

Figura 1. Diseño de Autonomía de Producción de Agua Potable. 


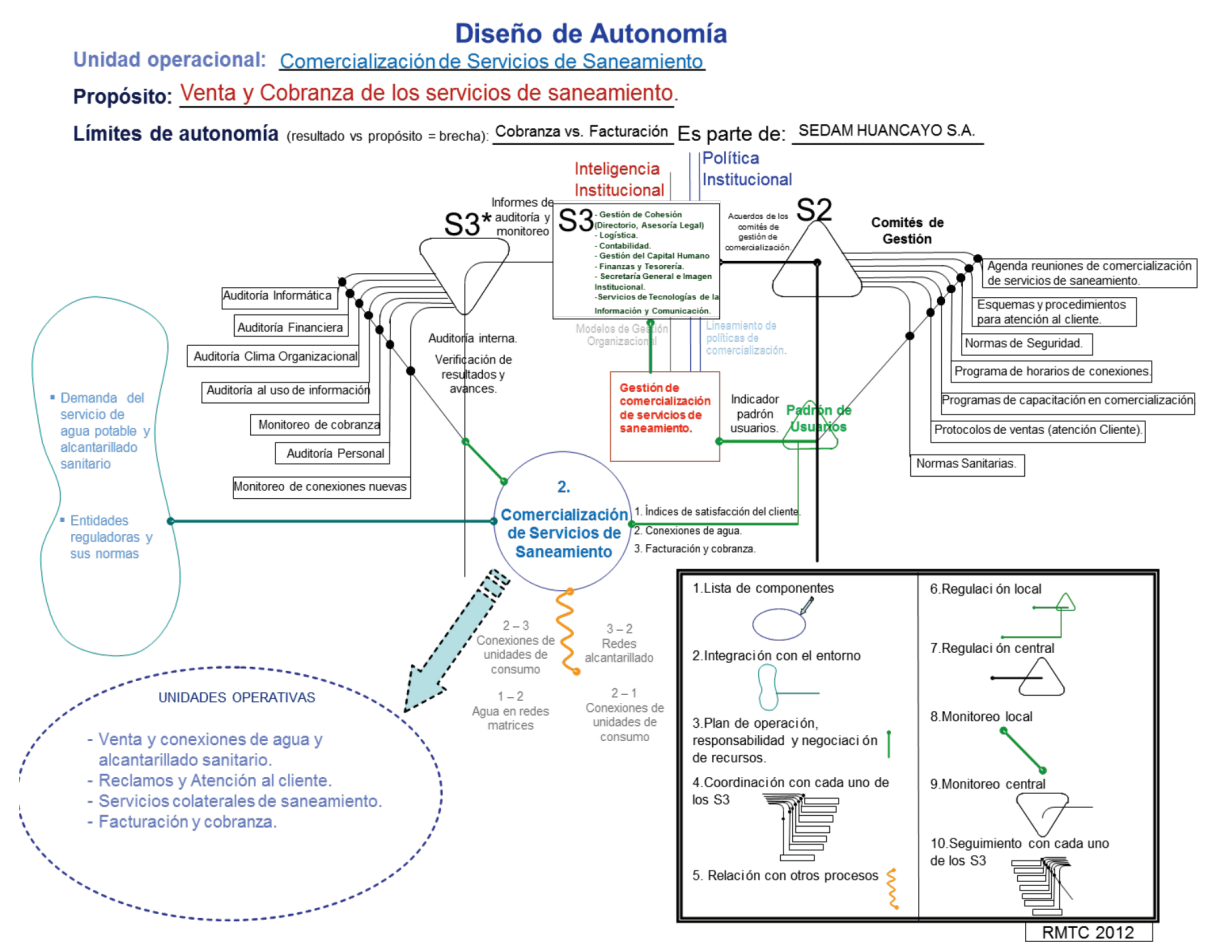

Figura 2. Diseño de Autonomía de Comercialización de Servicios de Saneamiento.

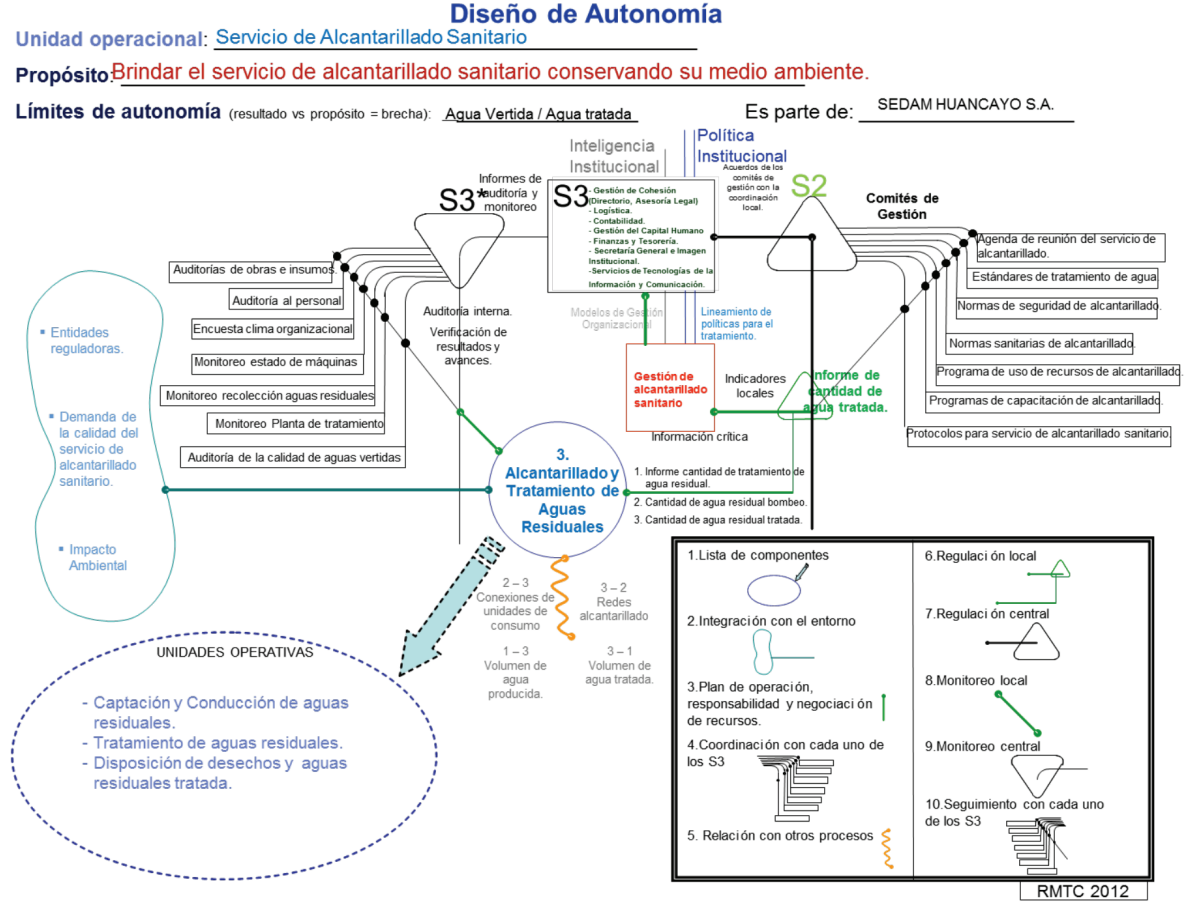

Figura 3. Diseño de Autonomía de Alcantarillado y Tratamiento de aguas residuales 


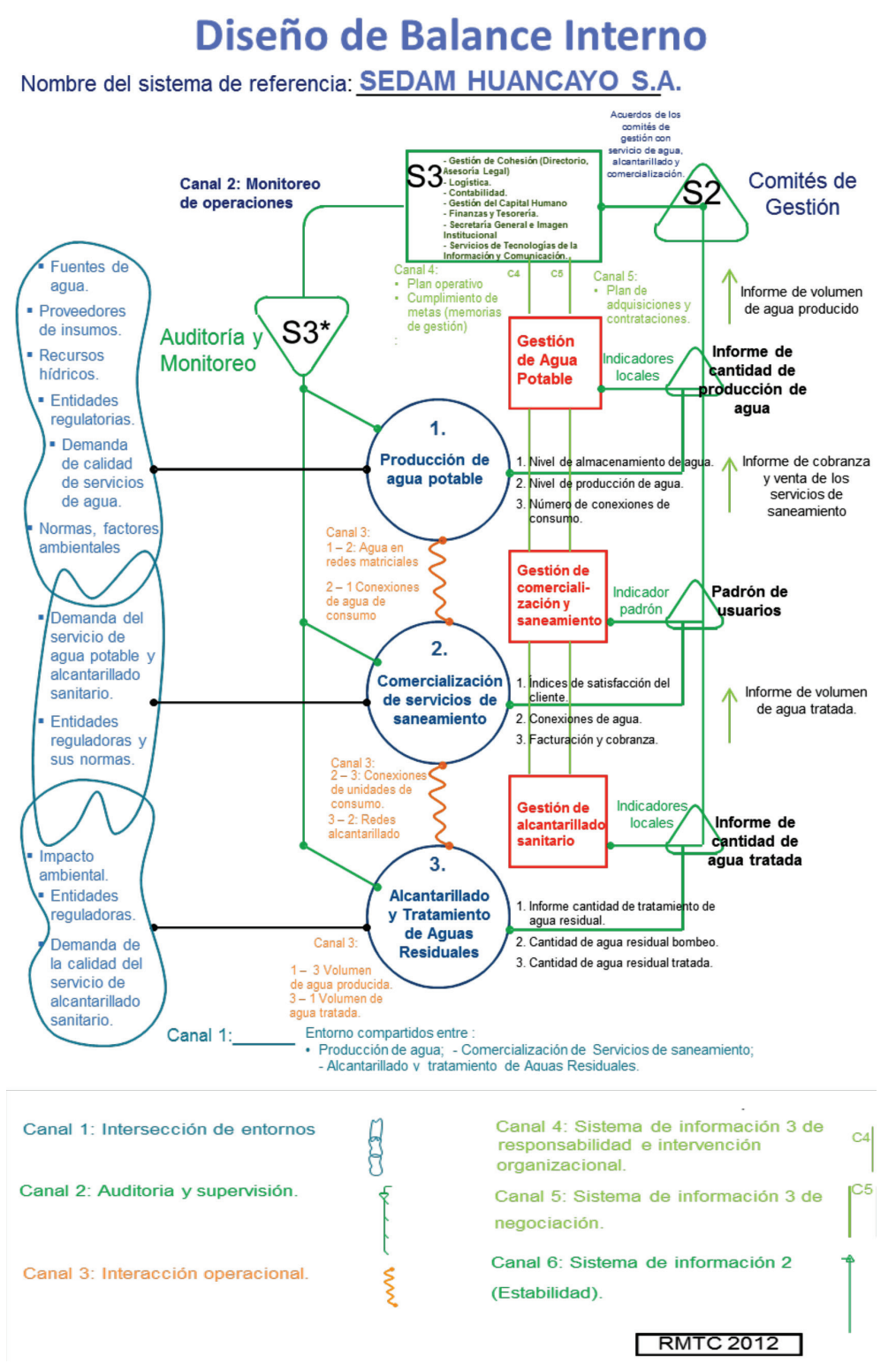

Figura 4. Diseño de Balance Interno de SEDAM HUANCAYO S.A.

- Cohesión: Logística, Gestión de Capital Humano, Contabilidad y Finanzas, Gestión de Servicios Tecnologías de la Información, Asesoría Legal, Secretaria General e Imagen Institucional, Gerencia de Cohesión (Directorio, Gestión de Cohesión y Sub Gestor de Cohesión).

- Monitoreo de operaciones: se realiza mediante la auditoría y monitoreo, el cual recoge información específica de acuerdo al proceso misional.

Luego continúa con el diseño del mecanismo de balance con el entorno de la empresa, es decir diseñar los procesos de adaptación e inteligencia, este mecanismo también es denominado "el afuera y el mañana". Los procesos de Planificación y prospectiva y Organización y mejora institucional son responsables del mecanismo de balance con el entorno, Figura 5.

Después, se diseña del mecanismo de identidad, ethos y política; este mecanismo está a cargo de Junta General de Accionistas, tres Gestores, uno por cada unidad misional, y el presidente del Directorio, Figura 6. 


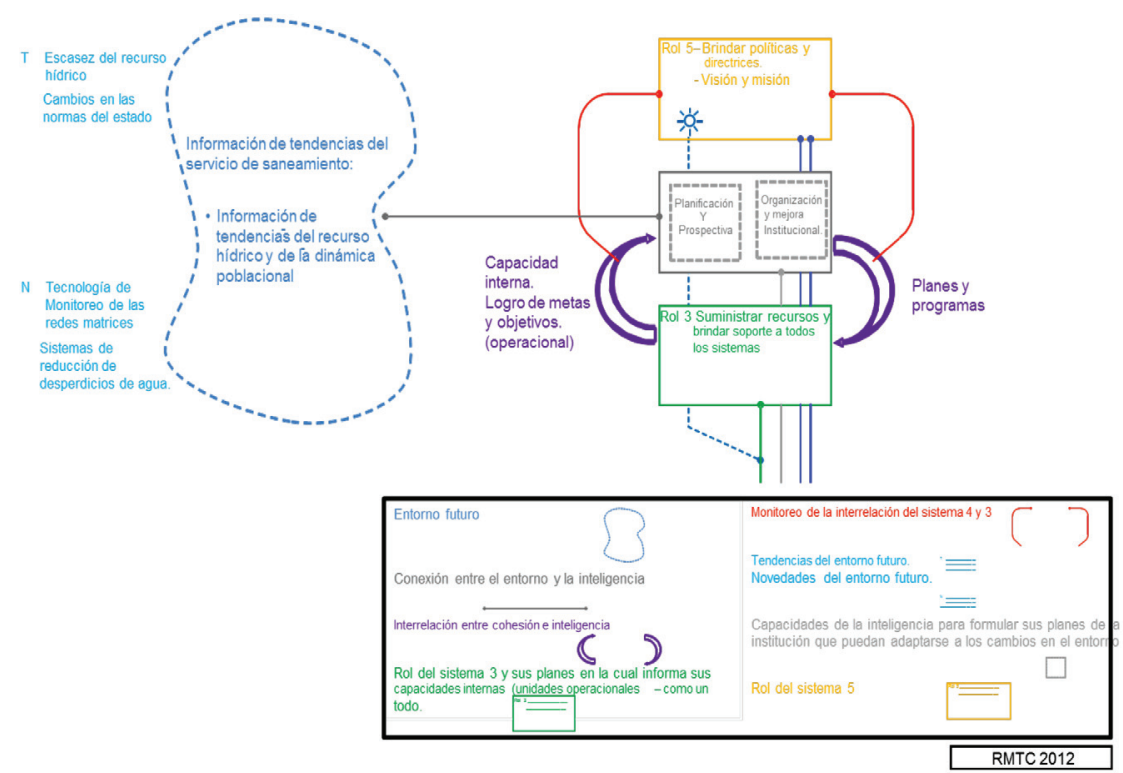

Figura 5. Diseño del Mecanismo de Balance con el entorno.
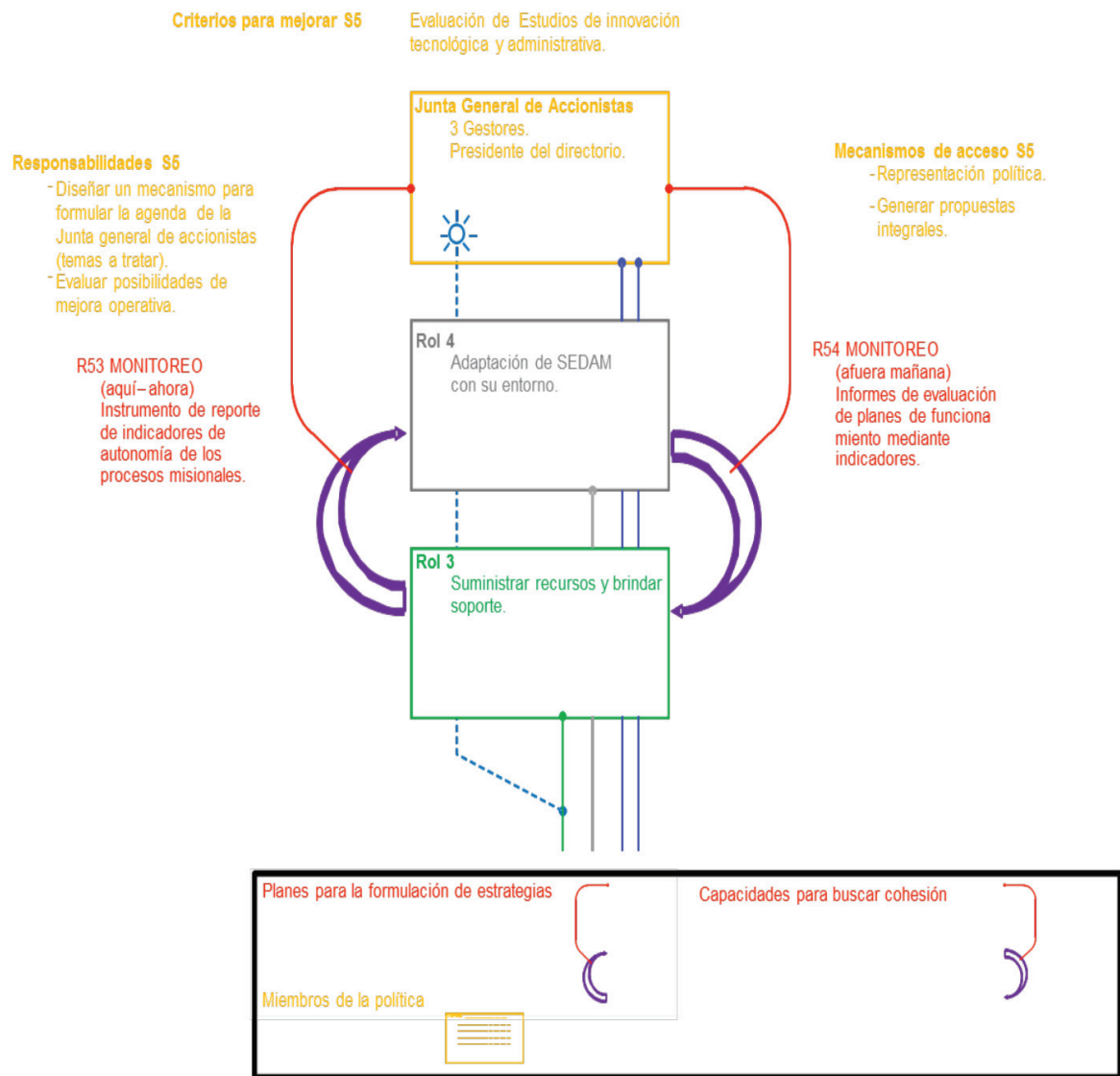

RMTC 2012

Figura 6. Diseño de Mecanismo de Política. 
Con el propósito de integrar el diseño organizacional, se desarrolla el modelo de sistema viable de la empresa en modo de diseño, eso permite representar y sintetizar las funciones sistémicas en la empresa, Figura 7.

Durante el diseño organizacional es preciso declarar la discrecionalidad en los procesos, es decir, el grado de centralización o descentralización funcional en la empresa. Para ello los procesos son agrupados en misionales y de gestión, Tabla 1.

Al desplegar las funciones sistémicas versus las actividades de regulación confirma la operación de las funciones sistémicas de viabilidad, estas son: política e identidad, inteligencia y adaptación, intervención corporativa, negociación de recursos, responsabilidad, monitoreo y seguimiento, estabilización y sincronización, Tabla 2.

Antes de culminar el diseño organizacional se desarrolla la definición de los macro procesos y procesos acorde a las funciones sistémicas de viabilidad y luego desplegar el mapa de procesos de la organización. El Macroproceso de SEDAM HUANCAYO S.A. viable se da de la siguiente manera:

- Se tiene como propósito: "Brindar servicio de saneamiento en su ámbito, en equilibrio con el medio ambiente".

- Para cumplir este propósito se debe contar con 6 macroprocesos: Misional, Estabilización y Sincronización, Cohesión, Monitoreo y Auditoría, Adaptación e Inteligencia e Identidad y Política.

- El Macroproceso Misional lo integran Producción de Agua Potable, Comercialización del Servicio de Saneamiento, Alcantarillado y Tratamiento de Aguas Residuales; el cual está encargado de llevar a cabo los procesos para cumplir el propósito de la institución.

- El Macroproceso de Estabilización y Sincronización está compuesto por Discutir Informes Misionales,

\section{SEDAM Huancayo S.A}

Propósito: Brindar el servicio de saneamiento en su ámbito, en equilibrio con el medio ambiente.

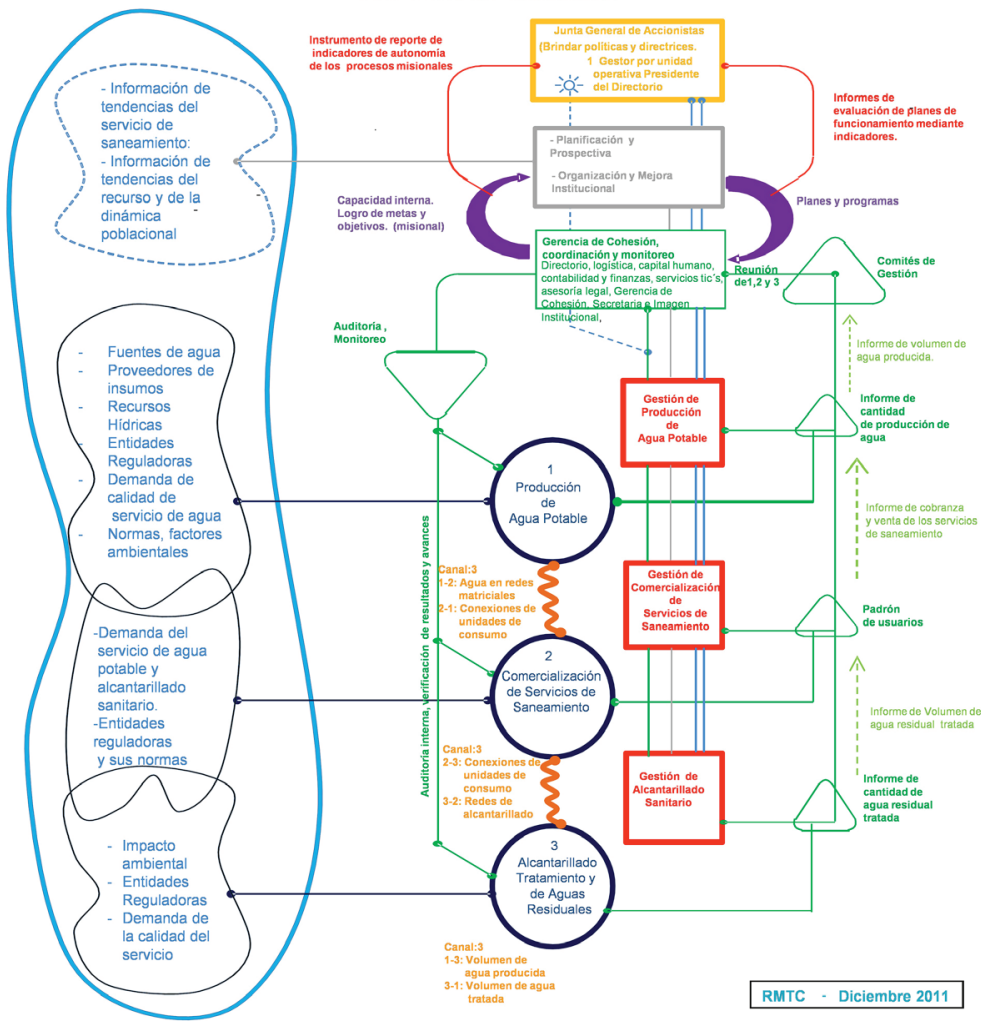

Figura 7. Modelo de Sistema Viable de SEDAM HUANCAYO S.A. 
Tabla 1. Análisis de Discrecionalidad.

\begin{tabular}{|c|c|c|c|c|c|c|c|c|c|c|c|}
\hline Gestión y soporte & 泡 & 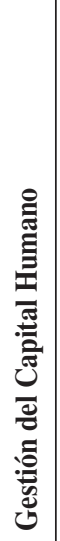 & 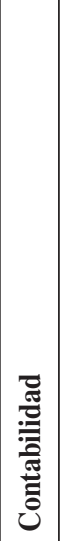 & 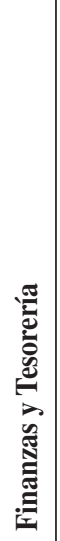 & 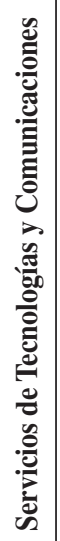 & 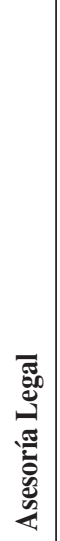 & 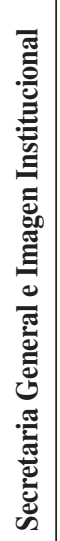 & 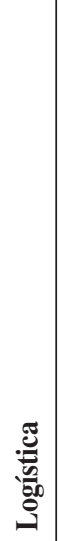 & 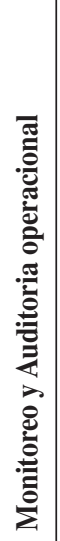 & 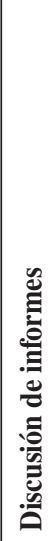 & 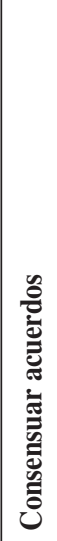 \\
\hline SEDAM HUANCAYO S.A. & o & $\mathrm{o}$ & $\mathrm{o}$ & $\mathrm{o}$ & $\mathrm{o}$ & o & $\mathrm{o}$ & $\mathrm{o}$ & o & o & o \\
\hline Producción de Agua Potable & $\mathrm{o}$ & & & & & & $\mathrm{o}$ & $\mathrm{o}$ & & $\mathrm{o}$ & $\mathrm{o}$ \\
\hline Almacenamiento y gestión de reserva de agua & & & & & & & $\mathrm{o}$ & $\mathrm{o}$ & & & \\
\hline Tratamiento de agua & & & & & o & & o & o & & & \\
\hline Distribución de agua potable & & & & & & & $\mathrm{o}$ & o & & & \\
\hline Comercialización de servicio de saneamiento & o & & & & & & $\mathrm{o}$ & $\mathrm{o}$ & & o & o \\
\hline Venta y conexiones de agua potable y alcantarillado sanitario & & & & & & & $\mathrm{o}$ & & & & \\
\hline Reclamos y atención al cliente & & & & & & & $\mathrm{o}$ & & & & \\
\hline Servicios colaterales de saneamiento & & & & & & & $\mathrm{o}$ & & & & \\
\hline Facturación y cobranza & & & & & $\mathrm{o}$ & & $\mathrm{o}$ & & & & \\
\hline Alcantarillado y Tratamiento de Aguas Residuales & o & & & & & & $\mathrm{o}$ & $\mathrm{o}$ & & o & o \\
\hline Captación y conducción de aguas residuales & & & & & & & o & o & & & \\
\hline Tratamiento de aguas residuales & & & & & $\mathrm{o}$ & & o & o & & & \\
\hline Disposición de desechos de aguas resi & & & & & & & $\mathrm{o}$ & $\mathrm{o}$ & & & \\
\hline
\end{tabular}

Tabla 2. Análisis de Funciones Sistémicas - Regulación.

\begin{tabular}{|c|c|c|c|c|c|c|c|c|c|c|c|c|c|c|c|}
\hline Funciones Sistémicas & 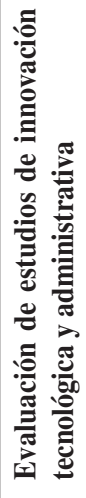 & 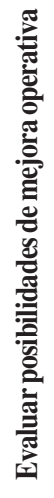 & 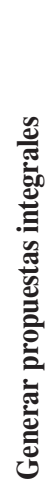 & 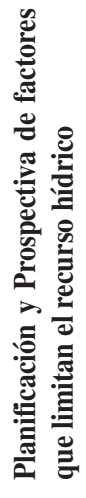 & 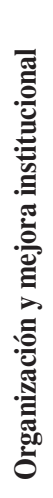 & 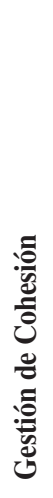 & 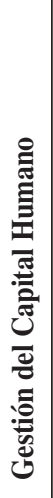 & 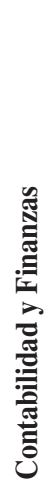 & 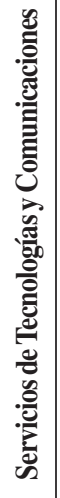 & 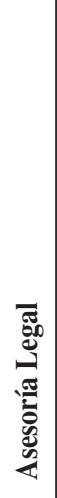 & 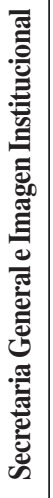 & 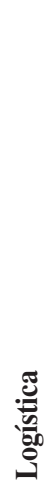 & 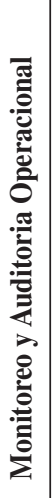 & 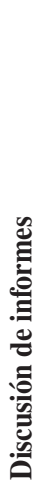 & 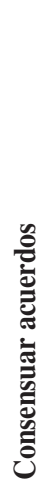 \\
\hline SEDAM HUANCAYO S.A. & $\mathrm{O}$ & $\mathrm{O}$ & $\mathrm{O}$ & $\mathrm{O}$ & $\mathrm{O}$ & $\mathrm{O}$ & $\mathrm{O}$ & $\mathrm{O}$ & $\mathrm{O}$ & $\mathrm{O}$ & $\mathrm{O}$ & $\mathrm{O}$ & $\mathrm{O}$ & $\mathrm{O}$ & $\mathrm{O}$ \\
\hline Política e Identidad & $\mathrm{O}$ & $\mathrm{O}$ & $\mathrm{O}$ & & & & & & & & & & & & \\
\hline Inteligencia y Adaptación & & & & $\mathrm{O}$ & $\mathrm{O}$ & & & & & & & & & & \\
\hline Intervención Corporativa & & & & & & $\mathrm{O}$ & & & & & & & & & \\
\hline Negociación de Recursos & & & & & & $\mathrm{O}$ & $\mathrm{O}$ & $\mathrm{O}$ & $\mathrm{O}$ & $\mathrm{O}$ & $\mathrm{O}$ & $\mathrm{O}$ & & & \\
\hline Responsablilidad & & & & & & $\mathrm{O}$ & & & & & & & & & \\
\hline Monitoreo y Seguimiento & & & & & & & & & & & & & $\mathrm{O}$ & & \\
\hline Estabilización y Sincronización & & & & & & & & & & & & & & 0 & \\
\hline
\end{tabular}


Consensuar Acuerdos Misionales y está encargado de mantener la comunicación efectiva entre los procesos misionales.

- El Macroproceso de Cohesión cuyos integrantes son: Gestión de Cohesión, Logística, Capital Humano, Contabilidad y Finanzas, Asesoría Legal, Secretaria e Imagen Institucional, Servicios en Tecnologías de la Información; es el encargado de la negociación de recursos que los procesos misionales requieren y son los encargados de recabar informes de responsabilidades, así como también tienen la función de Intervención corporativa.

- El Macroproceso de Monitoreo y Auditoría integrado por los procesos: Evaluar el Funcionamiento de Procesos Misionales, Brindar Recomendaciones para optimizar la integración de procesos misionales, está encargado de realizar auditorías y monitoreo de manera frecuente y no programado, además de informar a los medios formales con el fin de realizar recomendaciones para integrar los procesos misionales.

- El Macroproceso de Adaptación e Inteligencia que está integrado por: Planificación y Prospectiva, Organización y Mejora Institucional; es el encargado de velar por el entorno futuro y la mejora institucional, se preocupa por mantener un homeostato dentro del sistema junto con el Macroproceso de Cohesión.

- El Macroproceso de Identidad y Política está integrado por: Evaluación de Estudios de Innovación Tecnológica, Evaluación de posibilidades de Mejora Institucional, este Macroproceso está encargado de brindar las políticas de lineamiento a toda la institución, también tiene la potestad de intervenir en el Macroproceso Misional, siempre y cuando este incumpla con las políticas y lineamientos de la institución y como consecuencia no se podría llegar a cumplir el propósito para el cual ha sido creado y diseñada la institución.

Asimismo, el Mapa de Procesos Figura 8, es resultado de la integración de las etapas de identificación, diagnóstico y diseño, con la participación de cada uno de los integrantes de la empresa y la interpretación es de la siguiente manera:

- Lectura al propósito, que se entiende como el ADN de la empresa, la razón de ser y debe responder a la pregunta ¿Cómo para qué existe la empresa?

- Para lograr el propósito de la empresa se debe cumplir los 3 procesos misionales: proceso de Producción de Agua Potable (1), proceso de Comercialización de servicios de saneamiento (2) y proceso de Alcantarillado y Tratamiento de Aguas Residuales (3), el resultado del Macroproceso Misional son los servicios de agua y alcantarillado, así también el agua para el consumo agrícola y ganadera, teniendo presente la satisfacción del usuario, manteniendo siempre el equilibrio con el medio ambiente.

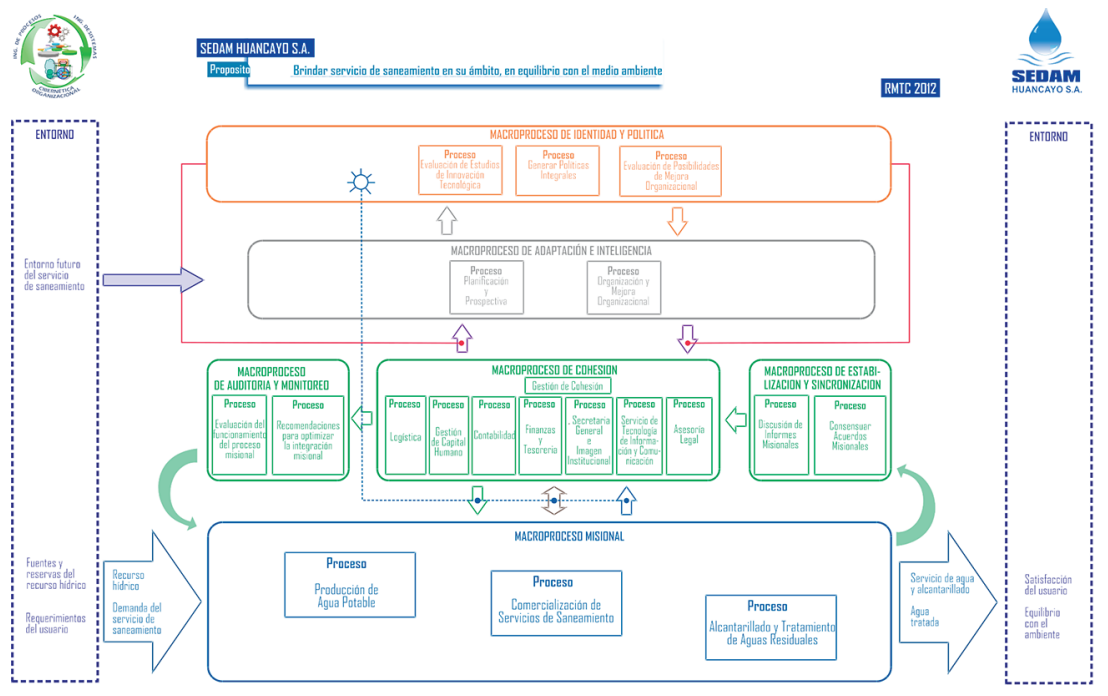

Figura 8. Mapa de Procesos de SEDAM HUANCAYO S.A. 


\section{DISCUSIÓN}

El diseño organizacional plantea una forma conveniente del cómo debería funcionar la empresa para desarrollarse en su medio, pensando en un sistema viable que permita obtener la flexibilidad necesaria para sobrevivir en ecosistemas rápidamente cambiantes y complejos. Para lo cual se ha considerado hacer un Diseño de Autonomía, que permita crear esencialmente las condiciones necesarias para todas las unidades misionales. También desarrollar un Equilibrio del Ambiente Interno, preocupado por las operaciones que se realizan en la empresa, y por último diseñar un Equilibrio con el Ambiente Externo que permita a los futuros sistemas de planificación desarrollar estrategias de adaptación a los cambios del mercado, sin dejar de lado el diseño de un mecanismo que brinde Identidad y Política de la Institución.

Por otro lado, el lenguaje de sistemas, un conjunto de términos y convenciones utilizadas de forma práctica y correcta en las metodologías sistémicas, en el presente caso de intervención está muy relacionada con la cibernética organizacional, en este punto es menester esclarecerlas y describirlas a fin de compartir un mismo contexto y significado de dichos términos y convenciones y estas son:

- Diseño de Autonomía, establece y afina el propósito de la organización, es decir su definición como sistema, detalla también sus componentes o unidades misionales. Se declara el entorno con el que interactúan estos componentes, es decir establece el requerimiento y el usuario, así como el resultado y el beneficiario. Límites de Autonomía: Esto está dado por un indicador que refleja la brecha generada por los resultados versus los propósitos y metas, este límite se activará en caso de no cumplir el propósito del sistema en general. Gestión Local: Es el responsable de llevar a cabo el plan de operación, de acuerdo a una correcta negociación de recursos con la alta dirección y a su vez interviene para hacer cumplir las reglas predefinidas. Coordinación Local: Son los informes y requerimientos que se obtiene como resultado de la ejecución de las operaciones [4], [25].

- Diseño de Balance Interno, Sistema 2 (Coordinación, Estabilización-Sincronización) son los instrumentos necesarios para la negociación de recursos (seguimiento local), que eviten inconvenientes para la unidad misional, como también eviten las oscilaciones innecesarias, que armonicen los esquemas de trabajo colectivo entre los diferentes grupos [25]. Sistema 3 (Cohesión), establece mecanismos de seguimiento local y también seguimiento central. Estos mecanismos no se implantan con la finalidad de sancionar o castigar a los procesos misionales, sino tienen el propósito de encontrar indicios de situaciones problemas. Sistema $3^{*}$ (auditoria y monitoreo), son reglas de intervención predefinidas (seguimiento central), servirán para conseguir información de diferente naturaleza que complementará, ampliará o validará la información que recibirá la administración por los demás canales de mando.

- Diseño del Balance con el Entorno, Sistema 4 (Adaptación e Inteligencia); al diseñar los procesos de adaptación e inteligencia es necesario que la empresa alcance óptimos resultados en el ahora y en su entorno relevante actual. Este mecanismo es el Sistema 4, también denominado el "Afuera y mañana" de la empresa SEDAM HUANCAYO S.A. consistente en la elaboración de planes a futuro. El entorno actual son todos aquellos aspectos relevantes, que están relacionados con el propósito del sistema, que forman parte del entorno. Para tener una idea más clara de lo que es el entorno.

- Diseño de mecanismos de Política, Sistema 5 (identidad y Política), el propósito del mecanismo de política es describir la identidad de la institución, y su funcionamiento para cambiar y transformarse continuamente así misma y lograr su viabilidad.

En el Perú, al menos cuatro EPS ya cuentan con un sistema de gestión de la calidad certificado de acuerdo con la Norma ISO 9001, [26], cuyo alcance comprende la totalidad de los procesos y actividades; algunas de ellas son: EMSA PUNO (2006) de 40000 usuarios, EPS MOQUEGUA (2009) de 19636 usuarios, EPS CHAVIN (2009) de 25000 usuarios, SEDA HUÁNUCO (2008) de 40345 usuarios [27].

La EPS SEDAM HUANCAYO S.A. de 65258 usuarios en el año 2012, a la fecha no posee ningún sistema de gestión de la calidad bajo ninguna norma, 
sin embargo, desde fines del año 2011 y comienzos del año 2012 a la fecha está utilizando el diseño organizacional mediante el enfoque sistémico y cibernético, y en todo momento, el diseño, influye en el fortalecimiento organizacional, mostrando crecimiento y mejora al igual de las EPS mencionadas.

Asimismo, el informe de benchmarking regulatorio, [27], muestra los resultados de la evaluación del desempeño de las 50 Empresas Prestadoras de Servicios de Saneamiento del Perú bajo el ámbito de regulación de la Superintendencia Nacional de Servicios de Saneamiento (SUNASS) cada año, demuestra que la empresa SEDAM HUANCAYO S.A. está mejorando, Figura 9.

Los indicadores utilizados del año 2007 hasta el año 2010 fueron: Cobertura de agua potable, Cobertura de alcantarillado, Micromedición, Porcentaje de conexiones activas, Porcentaje de tratamiento de aguas residuales, Contenido de cloro residual en redes, Relación de trabajo, Agua no facturada y Morosidad. A partir del año 2011 hasta el 2013, fueron utilizados 14 indicadores para el cálculo del benchmarking: Cobertura de agua potable, Cobertura de alcantarillado, Tiempo de atención de conexiones nuevas de agua potable, Porcentaje de tratamiento de aguas residuales, Índice de satisfacción del cliente, Índice de clima laboral, Agua no facturada, Relación de trabajo, Densidad de reclamos, Costo de energía eléctrica por volumen producido, Información en la página web de la EPS, Micromedición, Indicador de gestión del riesgo de desastres, Índice de cumplimiento global de las metas de gestión [27], Figura 9.

Por otro lado, el cálculo del benchmarking de los años 2010 al 2012, fue en base a 14 indicadores: Cobertura

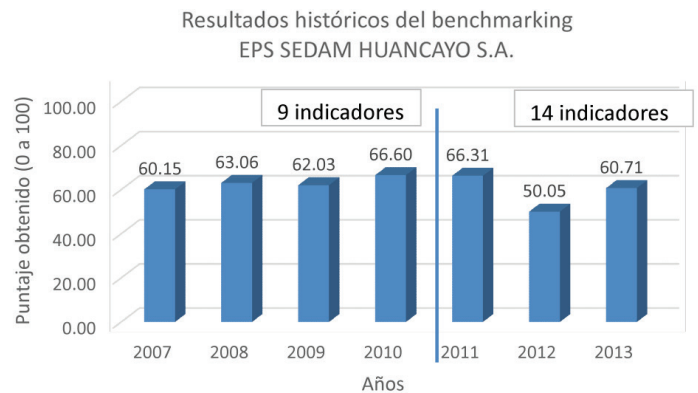

Figura 9. Resultados históricos del benchmarking regulatorio [27]. de agua potable, Cobertura de alcantarillado, Tiempo de atención de conexiones nuevas de agua potable, Porcentaje de tratamiento de aguas residuales, Índice de satisfacción del cliente, Índice de clima laboral, Densidad de roturas, Relación de trabajo, Densidad de atoros, Costo de energía eléctrica por volumen producido, Información en la página web de la EPS, Micromedición, Indicador de gestión del riesgo de desastres, Continuidad. Indicadores definidos inicialmente para medir el alineamiento de las EPS al cumplimiento de los objetivos del Plan Nacional de Saneamiento, para la evaluación del desempeño del año 2013, fueron 13 indicadores debido a que no se consideró el indicador de gestión del riesgo de desastres ya que menos del $30 \%$ de EPS tenía información al respecto, y para la evaluación del desempeño del año 2014, se volvieron a utilizar los 14 indicadores [28], Figura 10.

\section{CONCLUSIONES}

El caso de estudio es un proceso de aplicación sistémica y cibernética organizacional en el contexto del servicio público de saneamiento local, en especial el diseño organizativo. El caso propone una nueva metodología de diseño organizacional sistémico-cibernético complementada con el enfoque de gestión basada en procesos. Los componentes del sistema organizativo fueron el resultado del Diseño de Autonomía, Diseño de Balance Interno, Diseño del Balance con el Entorno y el Diseño de mecanismos de Política.

El diseño organizacional como una intervención sistémica y cibernética plantea una forma de operar de la empresa en su entorno, que le permite obtener la flexibilidad para sobrevivir en ecosistemas

Resultados históricos del benchmarking

EPS SEDAM HUANCAYO S.A.

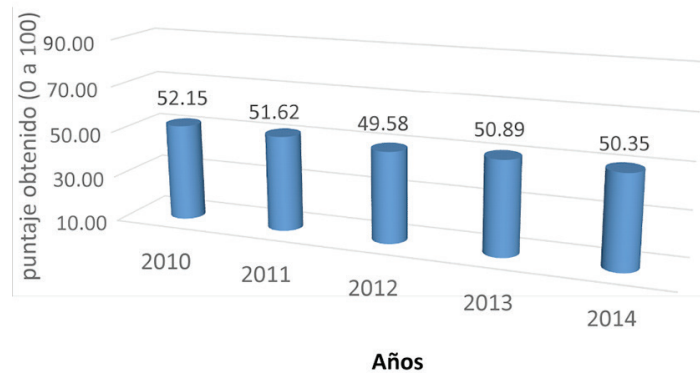

Figura 10. Resultados históricos del benchmarking 2014 [28]. 
cambiantes y complejos, además implementar su sistema organizativo mediante la gestión basada en procesos, es decir, sus procesos agrupados en funciones sistémicas.

El diseño organizacional involucro el cambio de puntaje en el benchmarking regulatorio 2013, de 50.05 en el año 2012 a 60.71 en el 2013, una situación sobresaliente. En el benchmarking regulatorio 2014, el puntaje varió de 49.58 en el año 2012, seguido por 50.89 el 2013 y de 50.35 el 2014, una situación deseada.

\section{AGRADECIMIENTOS}

A la EPS SEDAM HUANCAYO S.A. por el interés y apoyo mostrado en la ejecución del proyecto.

\section{REFERENCIAS}

[1] R. Noticias. "Problemas de saneamiento básico generan desnutrición y mortalidad infantil”. RPP Noticias, 9 Abril 2015. [En línea]. Available: http://rpp.pe/lima/ actualidad/problemas-de-saneamiento-basicogeneran-desnutricion-y-mortalidad-infantilnoticia-786163. Último acceso: 6 Junio 2015.

[2] F. Alayo Orbegozo. "El Comercio Sociedad", El Comercio, 6 Agosto 2016. Available: http://elcomercio.pe/sociedad/peru/ agua-pocos-saneamiento-pendiente-perunoticia-1922345. Último acceso: 22 Agosto 2016.

[3] S. Beer. "Brain of the Firm", Chichester: Wiley. 1981.

[4] S. Beer. "Diagnosing the System for Organizations". London: Wiley. 1985.

[5] J. Pérez Ríos. "Diseño y diagnóstico de organizaciones viables. Un enfoque sistémico", España: Iberfora 2000. 2008.

[6] P. Hoverstadt. "The Fractal Organization: Creating sustainable organizations with the Viable System Model". Australia: John Wiley \& Sons, Ltd. 2009.

[7] A. Reyes and R. Espejo. Organizational Systems. Managing Complexity with the Viable System Model, London: Springer. 2011.

[8] H. Nechansky. "Issues of organizational cybernetics and viability beyond Beer's viable". International Journal of General Systems. Vol. 42 No 8, pp. 838-859. 2013.
[9] J. Puche, B. Ponte and J. Costas. "Systemic approach to supply chain management through the viable system model", Production Planning \& Control. Vol. $27 \mathrm{~N}^{\mathrm{o}}$ 5, pp. 421430. 2016.

[10] J. Puche. "Extending the viable system model scope on ICT-sector software projects in Castilla y León”, Kybernetes. Vol. 43 N², pp. 192-209. 2014.

[11] J. Pérez Ríos and I. Velasco Jiménez. "The application of organizational cybernetics and ICT to collective discussion of complex issues". Kybernetes. Vol. 44 N $6 / 7$, pp. 11461166, 2015.

[12] K. Akmaliah Adham, H. Kasimin, R. Mat Isa, F. Othman and F. Ahmad. "Developing a Framework for a Viable Research". Systemic Practice and Action Research. Vol. $28 \mathrm{~N}^{\circ} 5$, pp. 503-525. 2015.

[13] S. Hildbrand and S. Bodhanya. "Guidance on applying the viable system model". Kybernetes. Vol. 44 No 2, pp. 186-201. 2015.

[14] J.C. Puche Regaliza. "Quantitative analysis of viable systems model on software projects in the ICT sector in Castilla y León". Kybernetes. Vol. $44 \mathrm{~N}^{\circ}$ 5, pp. 806-822. 2015.

[15] C. Gershenson. "Requisite variety, autopoiesis, and self-organization", Kybernetes. Vol. 44 No 6/7, pp. 866-873. 2015.

[16] M. Schwaninger. "Organizing for sustainability:a cybernetic concept forsustainable renewal". Kybernetes. Vol. $44 \mathrm{~N}^{\circ} 6 / 7$, pp. 935-954. 2015.

[17] A. Espinosa. "Governance for sustainability: learning from VSM practice". Kybernetes. Vol. $44 \mathrm{~N}^{\mathrm{o}}$ 6/7, pp. 955-969. 2015.

[18] B. Sagalovsky. "Organizing for Lean: autonomy, recursion and cohesion". Kybernetes. Vol. 44 No 6/7, pp. 970-983. 2015.

[19] J. Pérez Rios and I. Velasco Jiménez. "The application of organizational cybernetics and ICT to collective discussion of complex issues", Kybernetes. Vol. 44 No 6/7, pp. 11461166. 2015.

[20] L.A.A. Terra, C.A.A. Ventura, M.L. Medeiros and J.L. Passador. "Strategies for the Distribution of Power in Brazil: A Proposal from the Perspective of the Viable System Model (VSM)". Systems Research and Behavioral Science. Vol. $33 \mathrm{~N}^{\circ} 2$, p. 224234. 2016. 
[21] P. Parraguez, S. Eppinger and A. Maier., "Characterizing Design Process Interfaces as Organization Networks: Insights for Engineering Systems Management", Systems Engineering. Vol. $19 \mathrm{~N}^{\circ}$ 2, pp. 158-173. 2016.

[22] D. Lowe, L. Martingale and M. Yearworth. "Guiding interventions in a multi-organisationalcontext: combining the Viable System Model andHierarchical Process Modelling for use as a ProblemStructuring Method". Journal of the Operational Research Society. Vol. $67 \mathrm{~N}^{\circ}$ 12, pp. 1-15. 2016.

[23] M. Schwaninger and C. Scheef. "A Test of the Viable System Model: Theoretical Claim vs. Empirical Evidence", CYBERNETICS AND SYSTEMS: AN INTERNATIONAL JOURNAL. Vol. 47 Nº 7, pp. 544-569. 2016.
[24] M. Walker. "The Search for Viability: A practitioner's view of how the Viable Systems Model is helping transform English local government (and why it has pa ssedunrecognised)", Systems Research and Behavioral Science. Vol. $34 \mathrm{~N}^{\circ} 3$, pp. 313334. 2017.

[25] R.-M. Taipe Castro. Pensamiento sistémico en el sector público, Huancayo: UNCP. 2010.

[26] Programa de Agua Potable y Alcantarillado (PROAGUA), Manual ISO 9001Gestión de Calidad en EPS, Lima-Perú: D \& R Inversiones Gráficas S.A.C. 2010.

[27] SUNASS. "Benchmarking regulatorio de las EPS 2012". SUNASS, Lima. 2013.

[28] SUNASS. "Benchmarking regulatorio de las EPS 2014”, SUNASS, Lima-Perú. 2014. 